\title{
Women's receptivity to Fetal Alcohol Spectrum Disorders prevention approaches: A case study of two regions in Russia
}

\author{
Tatiana Balachova ${ }^{1}$, Barbara Bonner ${ }^{1}$, David Bard ${ }^{1}$, Mark Chaffin $^{1}$, Galina Isurina ${ }^{2}$, Arthur Owora ${ }^{1}$, Larissa \\ Tsvetkova ${ }^{2}$, and Elena Volkova ${ }^{3}$ \\ ${ }^{1}$ University of Oklahoma Health Sciences Center, Oklahoma City, Oklahoma, United States \\ ${ }^{2}$ St. Petersburg State University, St. Petersburg, Russia \\ ${ }^{3}$ Nizhny Novgorod State Pedagogical University, Nizhny Novgorod, Russia
}

\begin{abstract}
Aims: This study obtained data to inform the development of programs for prevention of Fetal Alcohol Spectrum Disorders (FASD) by examining Russian women's perceptions about the determinants of their decisions regarding alcohol consumption during pregnancy; the importance of educating professionals and community about FASD; and the credibility of various sources of information.
\end{abstract}

Design: Cross-sectional survey.

Setting: Seven women's clinics in St. Petersburg and the Nizhny Novgorod region in Russia.

Participants: Six hundred and forty-eight pregnant and non-pregnant women of childbearing age.

Measures: A face-to-face structured interview assessed demographic characteristics, pregnancy status, alcohol consumption, and level of trust in and receptivity to FASD prevention messages.

Findings: The most influential contributor to women's decisions regarding alcohol consumption during pregnancy was their own knowledge, followed by information from an obstetrician/gynecologist or nurse. It was most important to women that obstetrics and gynecology professionals and husbands or partners were knowledgeable about the effects of drinking during pregnancy. Physicians' recommendations and research data were regarded by the women as the most credible sources of information. There were significant variations in responses by socio-demographic characteristics and alcohol consumption levels. Younger women were more likely to report the contributions of husbands, mothers, and friends or coworkers to their decisions about alcohol consumption and indicated the importance of educating these people. Women at risk for alcohol use during pregnancy reported greater influence of husbands or partners and warning labels on containers on their alcohol consumption.

Conclusions: This study emphasizes the importance of broadly disseminating information about FASD, particularly research data, through education of health professionals and the general public in Russia. Women's socio-demographic characteristics and alcohol consumption levels should be considered in designing prevention programs.

There is growing evidence that prenatal alcohol exposure can lead to a wide range of Fetal Alcohol Spectrum Disorders (FASD) (American Academy of Pediatrics [AAP], 2000; Interagency Coordinating Committee on Fetal Alcohol Spectrum Disorders [ICCFASD], 2011; Warren, Hewitt, \& Thomas, 2011). Despite the fact that FASD are completely preventable, substantial numbers of women consume alcohol during pregnancy (Centers for Diseases Control and Prevention [CDC], 2012; Skagerstrom, Chang, \& Nilsen, 2011). An additional challenge is that approximately half of pregnancies are unplanned and many women consume alcohol at prepregnancy levels prior to knowledge of pregnancy (Ethen et al., 2007; Floyd, Decoufle, \& Hungerford, 1999).

The problem is more prevalent in countries with higher alcohol use and limited prevention programs, such as Russia. The rate of alcohol consumption in Russia is among the highest in the world (World Health Organization

Correspondence: Tatiana Balachova, Ph.D., University of Oklahoma Health Sciences Center, 1100 N.E. $13^{\text {th }}$ Street, Oklahoma City, OK 73117 United States. Telephone: (405) 271-8858. Fax: (405) 271-2931. E-mail: Tatiana-Balachova@ouhsc.edu

Financial support: This publication is supported by research grants R21TW006745 and R01AA016234 from the National Institutes of Health/National Institute on Alcohol Abuse and Alcoholism and Fogarty International Center (Brain Disorders in the Developing World: Research Across the Lifespan). The content is solely the responsibility of the authors and does not necessarily represent the official views of the National Institute on Alcohol Abuse and Alcoholism, Fogarty International Center, or the National Institutes of Health.

Keywords: Fetal Alcohol Spectrum Disorders, Russia, alcohol consumption, health message perceptions, prevention 
[WHO], 2011) and hazardous drinking among women is increasing (Onischenko, 2007). Nearly all Russian women report drinking before pregnancy; during pregnancy, depending on the study, $20 \%$ to $60 \%$ drink to some extent and $3 \%$ to $7.4 \%$ report binge drinking (Balachova et al., 2012; Chambers, Kavteladze, Joutchenko, Bakhireva, \& Jones, 2006; Kristjanson, Wilsnack, Zvartau, Tsoy, \& Novikov, 2007). Moreover, a significant portion of physicians and the general public in Russia still have only limited knowledge about the effects of alcohol consumption during pregnancy (Balachova, Bonner, Isurina, \& Tsvetkova, 2007). Although FAS rates in the Russian general population have not been established, studies report high FASD rates in Russian orphanages (Miller et al., 2006; Palchik, Legonkova, \& Sofronova, 2011; Riley et al., 2003) and in children adopted from Russia (Landgren, Svensson, Stromland, \& Andersson Gronlund, 2010). FASD constitutes a significant problem for public health and therefore warrants increased attention and prevention efforts (Barry et al., 2009; Hankin, 2002).

Several public health strategies to prevent FASD have been developed and implemented in Western countries, including public awareness campaigns, screenings and brief interventions in primary care, community interventions, and other approaches (Floyd, Weber, Denny, \& O'Connor, 2009; Hankin, 2002; Poole, 2008; Rasmussen et al., 2012). These efforts have shown some efficacy in reducing rates of alcohol use among pregnant women (CDC, 2012; Nilsen, Holmqvist, Hultgren, Bendtsen, \& Cedergren, 2008). However, there is a growing recognition of the link between the context of health communication and intervention effectiveness which has underscored the need for culturally congruent health message delivery. As indicated by the American Public Health Association (Lum, Parvanta, Maibach, Arkin, \& Nelson, 2002, p. 48), communicating health information effectively requires two components: (1) determining what is needed in prevention approaches and content, and (2) providing what is needed in a way that can be understood and used by the audience, with emphasis on evidence-informed strategies. As a result of the attention paid to evidence-based interventions, there is increasing interest in the application of social marketing principles to health behavior research (Kotler \& Lee, 2008) and an understanding that prevention approaches need to be responsive to the target audience's receptivity to prevention messages (Howat et al., 2003; Howat, Sleet, Elder, \& Maycock, 2004). A prerequisite for social marketing interventions is formative research to better understand how target audiences and individuals may react to different messaging contexts (Atkin \& Smith, 2010; Siegel \& Lotenberg, 2007).

In the field of FASD prevention, few studies have focused on research examining perceptions of widely used messaging contexts and approaches (Foundation for Alcohol Research and Education [FARE], 2011; Yu, Ahern, Connolly-Ahern, \& Shen, 2010). Data about women's receptivity to different sources of information continue to be limited, and information about Russian women's perceptions and preferences in prevention is unavailable. The objective of the current study was to answer the following research questions: Among women in Russia, (1) what or who is perceived as an influential determinant of whether or not alcohol is consumed during pregnancy? (2) whose education about alcohol use during pregnancy and FASD is perceived as most important? and (3) what is the credibility of various prevention message delivery modes used in health promotion?

\section{Method}

\section{Setting and Sample}

The data used in this analysis were gathered as part of a larger formative study designed to evaluate pregnant and non-pregnant women's alcohol consumption, knowledge, and attitudes related to alcohol consumption during pregnancy for the purpose of developing FASD prevention programs in Russia. Participants were women of childbearing age (18-44 years) recruited at seven public women's clinics in St. Petersburg (SPB) and in the Nizhny Novgorod Region (NNR), representing urban (SPB) and more rural (NNR) areas. In Russia, public women's clinics provide routine obstetrics and gynecology services, family planning, and prenatal and postpartum care at no charge for women residing in areas served by the clinics. As well as providing medical care, the clinics issue the formal documents required for maternity leave and benefits. Government statistics indicate that $96.4 \%$ of pregnant women in Russia receive prenatal services from public women's clinics (Sukhanova, 2008). Therefore, a representative sample of women of childbearing age can be recruited though these clinics in Russia.

Recruitment was stratified; pregnant and non-pregnant women were recruited as consecutively enrolled patients at both study locations. Prospective participants were approached in clinic waiting rooms and approximately $80 \%$ of women approached in SPB and $89 \%$ in NNR agreed to participate. Study procedures were conducted in private face-to-face interviews by local, trained research staff members who were female graduate students familiar with the language and customs of the area. A total of 657 women were enrolled in the study; nine were excluded due to age or incomplete information, resulting in a total sample of 648, including 342 women from SPB (146 pregnant, 196 non-pregnant) and 306 women from NNR (155 pregnant, 151 non-pregnant) (Table 1). The study was reviewed and approved by IRBs at St. Petersburg State University and the University of Oklahoma Health Sciences Center and was conducted with approvals from the participating clinics.

\section{Measures}

Data were collected using a structured 40- to 50-minute interview. Survey items were constructed based on findings from previous Russian studies (Balachova et al, 2007) and extensively-researched U.S. and international measures (Kaskutas, 2000; Kesmodel \& Olsen, 2001; Russell, 1994; Sokol, Martier, \& Ager, 1989). Questions were reviewed for cultural appropriateness and then underwent standard forward and backward translation procedures and piloting before implementation. During 
pilot testing, women indicated that they preferred face-toface interviews over a group interview or self-administered measures. The survey gathered information about women's demographic characteristics, alcohol consumption, attitudes concerning drinking during pregnancy, receptivity to FASD prevention programs, and social influences on health beliefs and behavior.

Attitudes, perceptions, and preferences questions. Each study participant was asked to rate (1) how various sources of information contributed, or would have contributed, to her decision regarding alcohol consumption during pregnancy (Table 2); (2) how important it was that various health professionals and people in the community receive information on drinking during pregnancy to help her in making a decision, and/or to support her decision, about drinking during pregnancy (Table 3); and (3) what kinds of information on drinking during pregnancy she finds credible, and how much she trusts this information (Table 4). All response scale ratings were based on a five-point Likert scale of importance (from Not at all to Very much/A lot).

Alcohol consumption measures. Due to the lack of health guidelines regarding alcohol consumption in Russia, U.S. standard drink definitions and recommended drinking limits were utilized. Risky drinking for non-pregnant women was defined as consuming eight or more drinks in a week or binge drinking (National Institute on Alcohol Abuse and Alcoholism [NIAAA], 2004). Non-pregnant women reported their alcohol consumption during the last three months, while pregnant participants reported their level of consumption in the three months before pregnancy and after recognizing pregnancy.

Quantity/frequency measure. Similar to beverage and container-specific approaches that have been used in other countries (Kesmodel, Kesmodel, Larsen, \& Secher, 2003; Kristjanson et al., 2007), a beverage-specific weekly quantity/frequency approach was used to determine standardized alcohol content and volume, which were then transformed to U.S. standard drink units (NIAAA, 2005).

Binge drinking. Women were asked how often they had consumed four or more drinks on a single occasion during the last three months. A binary indicator of binge drinking was used in the analyses; that is, any binge drinking was taken to constitute risky drinking (NIAAA, 2004).

Screening for risk of alcohol consumption during pregnancy. Pregnant and non-pregnant women completed the T-ACE (Sokol et al., 1989) and TWEAK (Russell, 1994) screening measures for risk of alcohol consumption during pregnancy.

\section{Statistical Analysis}

Univariate and bivariate analyses were used to describe the general characteristics of the study sample stratified by pregnancy status and city. The proportion distribution of women's response ratings (ordered responses) were compared across groups defined by pregnancy status and city using a non-parametric Jonckheere-Terpstra (JT) test (Sprent, 2001). Multinomial logistic regression was used to model the nominal response ratings regarding perceptions of, trust in and receptivity to various prevention approaches. Specifically, log odds of the rating responses (outcomes) were modeled as a linear combination of predictor variables. Selected predictor/explanatory variables (all binary) included indicators of binge drinking; drinking in excess of weekly limits; risk for alcohol consumption during pregnancy (T-ACE and TWEAK); and socio-demographic variables including marital status, education level, age, and income. To satisfy sample size requirements at each response level in the analyses, we collapsed the five-point Likert scale by merging response options Not at all, Not very much, and Not sure into a single level to form three level outcome variables (Not at all/Not very much/Not sure-0, Somewhat-1, and A lot or Very much-2) for the three questions of interest (Tables 2, 3 , and 4). Statistical significance was set at $p<.05$ and all analyses were performed using SAS, version 9.1.3, software (SAS Institute, Inc., 2008).

\section{Results}

\section{Sample descriptive information}

The average age of non-pregnant women was 28.9 years of age. The average pregnant interviewee was 27.5 years old and at $20.6(S D=9.0)$ weeks of gestation. A larger proportion of SBP participants had higher education, higher income, and more urban residence than in NNR, all comparable to expectations based on the regional demographic characteristics reported by the Russian Federation Federal State Statistics Service (2009). A summary of study participants' demographic information is presented in Table 1.

\section{Influential determinants/contributors to decisions regarding alcohol use during pregnancy}

Influential contributors to women's decisions regarding alcohol use during pregnancy are reported in Table 2. For conciseness, only significant main effects are described in this section. The determinant with the greatest potential to influence a woman's decision regarding alcohol consumption during pregnancy was her own knowledge (A lot or Somewhat-70\%; 95\% CI: 63-77), closely followed by an obstetrician/gynecologist (OB/GYN) or nurse (A lot or Somewhat-68\%; 95\% CI: 61-75), and then by her husband or partner (A lot or Somewhat-60\%; 95\% CI: 5367). Pregnant women were more likely to select their own knowledge as an influential determinant of their alcohol consumption during pregnancy than non-pregnant women, adjusting for the city of residence (A lot vs. Not at all/Not very much: Odds ratio: 1.71; 95\% CI: 1.11-2.64 and Somewhat vs. Not at all/Not very much: Odds ratio: 1.01; 95\% CI: 0.62-1.64). 
Table 1

Demographic characteristics of sample $(N=648)^{a}$

\begin{tabular}{|c|c|c|c|c|c|}
\hline \multirow[b]{3}{*}{ Demographic category } & \multicolumn{5}{|c|}{ Frequency (Percentage) } \\
\hline & \multicolumn{2}{|c|}{ Pregnant } & \multicolumn{2}{|c|}{ Non-pregnant } & \multirow[b]{2}{*}{ Total $(N=648)$} \\
\hline & $\begin{array}{c}\mathrm{SPB}_{1} \\
(N=146)\end{array}$ & $\begin{array}{c}\mathrm{NNR}_{2} \\
(N=155)\end{array}$ & $\begin{array}{c}\mathrm{SPB}_{3} \\
(N=196)\end{array}$ & $\begin{array}{c}\mathrm{NNR}_{4} \\
(N=151)\end{array}$ & \\
\hline Average age in years $(S D)$ & $26.9(4.8)_{4}$ & $28.1(5.6)_{4}$ & $26.5(6.3)_{4}$ & $31.9(6.5)_{1,2,3}$ & $28.2(6.2)$ \\
\hline Age range & {$[18.4-40.9]$} & {$[18.2-44.0]$} & [18.0-43.5] & {$[18.1-43.9]$} & {$[18.0-44.0]$} \\
\hline \multicolumn{6}{|l|}{ Marital status } \\
\hline Married & $98(67.1 \%)_{3,4}$ & $124(80.0 \%)_{3,4}$ & $65(33.2 \%)_{1,2}$ & $63(41.7 \%)_{1,2}$ & $350(54.0 \%)$ \\
\hline Cohabitating & $34(23.3 \%)$ & $16(10.3 \%)$ & $34(17.3 \%)$ & $32(21.2 \%)$ & $116(17.9 \%)$ \\
\hline Single/divorced ${ }^{\mathrm{b}}$ & $14(9.6 \%)$ & $15(9.7 \%)$ & $96(49.0 \%)$ & $56(37.1 \%)$ & $181(27.9 \%)$ \\
\hline \multicolumn{6}{|l|}{ Living environment $^{c}$} \\
\hline Urban inner city & $141(96.6 \%)$ & $84(54.2 \%)_{3}$ & $190(96.9 \%)_{2,4}$ & $95(62.9 \%)_{3}$ & $510(78.7 \%)$ \\
\hline \multicolumn{6}{|l|}{ Education } \\
\hline No school diploma & $0(0.0 \%)_{2,4}$ & $4(2.6 \%)_{1,3}$ & $2(1.0 \%)_{2,4}$ & $3(1.2 \%)_{1,3}$ & $9(1.4 \%)$ \\
\hline School diploma & 17 (11.6\%) & $91(58.7 \%)$ & 34 (17.3\%) & $93(61.6 \%)$ & 235 (36.3\%) \\
\hline Middle level college & $61(41.8 \%)_{2,4}$ & $5(3.3 \%)_{1,3}$ & $72(36.8 \%)_{2,4}$ & $1(0.7 \%)_{1,3}$ & $139(21.4 \%)$ \\
\hline Higher education/Ph.D. & $68(46.6 \%)$ & $55(35.5 \%)$ & 87 (44.4\%) & $54(35.7 \%)$ & $264(40.7 \%)$ \\
\hline \multicolumn{6}{|l|}{ Employment $^{\mathrm{d}}$} \\
\hline Employed & $81(55.5 \%)_{2,3,4}$ & $129(83.2 \%)_{13}$ & $130(66.3 \%)_{1,2,4}$ & $130(86.1 \%)_{1,3}$ & $470(72.5 \%)$ \\
\hline Student & $11(7.5 \%)$ & $6(3.9 \%)$ & $41(20.9 \%)$ & $11(7.3 \%)$ & $69(10.7 \%)$ \\
\hline Homemaker & $18(12.3 \%)$ & $13(8.4 \%)$ & $9(4.6 \%)$ & $5(3.3 \%)$ & $45(6.9 \%)$ \\
\hline Maternity leave & $23(15.8 \%)$ & $0(0.0 \%)$ & $9(4.6 \%)$ & $1(0.7 \%)$ & $33(5.1 \%)$ \\
\hline Unemployed/disability & $13(8.9 \%)$ & $7(4.5 \%)$ & $5(2.6 \%)$ & $4(2.6 \%)$ & $29(4.5 \%)$ \\
\hline 3,000-5,000 rubles & $51(34.9 \%)$ & $61(39.4 \%)$ & $61(31.1 \%)$ & $51(33.8 \%)$ & 224 (34.6\%) \\
\hline $5,000-10,000$ rubles & $55(37.7 \%)$ & $30(19.4 \%)$ & $74(37.8 \%)$ & $11(7.3 \%)$ & $170(26.2 \%)$ \\
\hline 10,000-18,000 > rubles & $30(20.5 \%)$ & $7(4.5 \%)$ & $30(15.3 \%)$ & $9(6.0 \%)$ & $76(11.7 \%)$ \\
\hline Missing $^{\mathrm{f}}$ & $2(1.4 \%)$ & $9(5.8 \%)$ & $10(5.1 \%)$ & $1(0.7 \%)$ & $22(3.4 \%)$ \\
\hline
\end{tabular}

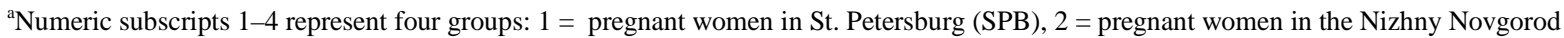
region (NNR), 3 = non-pregnant women in SPB, 4 = non-pregnant women in NNR. Subscripts in the table indicate which groups are significantly different $(\alpha$ set $=0.05)$ based on Bonferroni-adjusted Multiple Comparisons between the four groups. For example, the subscript shown on the column labeled "Pregnant $\left(\mathrm{SBP}_{1}\right)$ " and row “Average age in years (SD)" with the figure $26.9(4.8)_{4}$ means there is a significant difference in the average age of pregnant women in SBP versus non-pregnant women in NNR.

bingle/divorced category also included separated and widowed.

${ }^{\text {c}}$ The bottom three categories (rural, small city, suburban) were collapsed and logistic regression was performed to assess group differences in proportion of inner-city participant residents.

${ }^{\mathrm{d}}$ The bottom four categories were collapsed and logistic regression was performed to assess group differences in proportion gainfully employed.

${ }^{\text {e } B e t w e e n ~ S e p t e m b e r ~} 2004$ and May 2005, exchange rates were 27.5 to 29.2 rubles per U.S. dollar.

${ }^{\mathrm{f}}$ Missingness is suppressed for all but the income variable, due to minimal non-response (non-response ranged from 0 to 4 women on all other variables).

Overall, a higher education level was significantly associated with a higher rate of responsiveness to all contributing people (OB/GYN or nurse, husband or partner, mother, and friends), compared to a lower education $\left(\chi^{2}[2\right.$, $N=631]=8.38, p<.05)$. Women with a higher education level also reported a greater role played by the price of alcoholic drinks ("too expensive") in influencing their alcohol use during pregnancy than women with a lower level of education $\left(\chi^{2}[2, N=596]=9.18, p<.05\right)$.

Women at risk of drinking during pregnancy, as indicated by T-ACE/TWEAK, reported a greater role (contribution) played by their husband or partner $\left(\chi^{2}[2, N=626]=10.11\right.$, $p<.05)$ and by warning labels $\left(\chi^{2}[2, N=596]=6.52, p<\right.$ 
.05) in influencing their consumption. Younger women (ages 18-29) were more likely to report that a significant contribution in determining their alcohol consumption was made by a husband or partner $\left(\chi^{2}[2, N=626]=10.04, p<\right.$ $.05)$, mother $\left(\chi^{2}[2, N=607]=16.90, p<.05\right)$, or a friend or coworker $\left(\chi^{2}[2, N=570]=8.56, p<.05\right)$.

\section{Importance of educating health professionals and community}

Responses about the importance of educating health professionals and the community are included in Table 3. Overall, of the people who might help them in making their own decisions, women rated it most important that OB/GYN physicians and nurses receive information on the effects of drinking during pregnancy (A lot or Somewhat 68\%; 95\% CI: 61-75), followed by their husband or partner
(A lot or Somewhat 63\%; 95\% CI: 56-70). There were significant differences in response ratings by pregnancy status and city with respect to the importance of an $\mathrm{OB} / \mathrm{GYN}$ or nurse, husband or partner, mother, and friend or coworker receiving information related to drinking during pregnancy $(p<.05)$. Women 30 years or older and at a higher risk of alcohol drinking during pregnancy (as indicated by T-ACE/TWEAK) assigned greater importance to $\mathrm{OB} / \mathrm{GYNs}$ and nurses having this information than did younger (ages 18-29) and lower-risk women $\left(\chi^{2}[2, N=\right.$ $628]=5.42, p=.07)$. Younger women were more likely to attribute $A$ lot (Odds ratio: 0.41; 95\% CI: $0.25-0.66$ ) or Somewhat (Odds ratio: 0.73 ; 95\% CI: $0.44-1.23$ ) to the importance of educating husbands or partners about alcohol use during pregnancy than were women 30 years and older.

Table 2

Determinants in making decisions regarding drinking during pregnancy: Proportions of study participants' responses across four groups defined by pregnancy status and city of residence

\begin{tabular}{|c|c|c|c|c|c|c|}
\hline \multirow{2}{*}{$\begin{array}{l}\text { How much have the following contributed to } \\
\text { your decision on whether to drink during } \\
\text { pregnancy, or how much would they have } \\
\text { contributed to your decision on whether to } \\
\text { drink during pregnancy if they had discussed } \\
\text { it with you? }\end{array}$} & $\begin{array}{c}\text { Full } \\
\text { Sample }\end{array}$ & \multicolumn{2}{|c|}{ SPB } & \multicolumn{2}{|c|}{ NNR } & \multirow{2}{*}{$\begin{array}{c}\text { Strata } \\
\text { Difference } \\
\\
\\
J T^{\pi} \\
\text { (Z Statistic) }\end{array}$} \\
\hline & Proportion & Pregnant & $\begin{array}{c}\text { Non- } \\
\text { pregnant }\end{array}$ & Pregnant & $\begin{array}{c}\text { Non- } \\
\text { pregnant }\end{array}$ & \\
\hline Husband/partner ${ }^{\mathrm{a}, \mathrm{b}, \mathrm{c}, \mathrm{z}, \mathrm{s}, \mathrm{e}}$ & & & & & & $-9.29 * *$ \\
\hline Not at all/Not very much/Not sure & 0.40 & 0.17 & 0.23 & 0.64 & 0.62 & \\
\hline Somewhat & 0.21 & 0.25 & 0.40 & 0.05 & 0.14 & \\
\hline A lot & 0.39 & 0.58 & 0.37 & 0.31 & 0.25 & \\
\hline \multicolumn{7}{|l|}{ Best female friend } \\
\hline Not at all/Not very much/Not sure & 0.59 & 0.28 & 0.38 & 0.88 & 0.84 & \\
\hline Somewhat & 0.27 & 0.42 & 0.47 & 0.07 & 0.11 & \\
\hline A lot & 0.14 & 0.30 & 0.15 & 0.05 & 0.05 & \\
\hline Mother $^{\mathrm{b}, \mathrm{c} 3, \mathrm{e}}$ & & & & & & $-9.79 * *$ \\
\hline Not at all/Not very much/Not sure & 0.45 & 0.17 & 0.31 & 0.75 & 0.62 & \\
\hline Somewhat & 0.19 & 0.26 & 0.33 & 0.07 & 0.12 & \\
\hline A lot & 0.35 & 0.58 & 0.36 & 0.18 & 0.26 & \\
\hline \multicolumn{7}{|l|}{ Father } \\
\hline Not at all/Not very much/Not sure & 0.58 & 0.33 & 0.48 & 0.83 & 0.71 & \\
\hline Somewhat & 0.16 & 0.24 & 0.28 & 0.04 & 0.09 & \\
\hline A lot & 0.26 & 0.43 & 0.24 & 0.13 & 0.20 & \\
\hline \multicolumn{7}{|l|}{ Grandmother } \\
\hline Not at all/Not very much/Not sure & 0.74 & 0.49 & 0.68 & 0.95 & 0.87 & \\
\hline Somewhat & 0.13 & 0.27 & 0.19 & 0.03 & 0.03 & \\
\hline A lot & 0.13 & 0.24 & 0.13 & 0.03 & 0.10 & \\
\hline \multicolumn{7}{|l|}{ Grandfather } \\
\hline Not at all/Not very much/Not sure & 0.77 & 0.53 & 0.71 & 0.96 & 0.89 & \\
\hline Somewhat & 0.12 & 0.26 & 0.17 & 0.01 & 0.02 & \\
\hline A lot & 0.11 & 0.21 & 0.12 & 0.03 & 0.09 & \\
\hline Friends/co-workers ${ }^{\mathrm{b}, \mathrm{e}}$ & & & & & & $-9.77^{* *}$ \\
\hline Not at all/Not very much/Not sure & 0.71 & 0.46 & 0.59 & 0.92 & 0.89 & \\
\hline Somewhat & 0.23 & 0.41 & 0.35 & 0.05 & 0.09 & \\
\hline A lot & 0.06 & 0.13 & 0.06 & 0.03 & 0.02 & \\
\hline \multicolumn{7}{|l|}{ Physician or nurse } \\
\hline Not at all/Not very much/Not sure & 0.48 & 0.12 & 0.35 & 0.70 & 0.82 & \\
\hline Somewhat & 0.22 & 0.35 & 0.31 & 0.12 & 0.08 & \\
\hline A lot & 0.30 & 0.53 & 0.34 & 0.18 & 0.11 & \\
\hline
\end{tabular}




\begin{tabular}{|c|c|c|c|c|c|c|}
\hline \multirow{2}{*}{$\begin{array}{l}\text { How much have the following contributed to } \\
\text { your decision on whether to drink during } \\
\text { pregnancy, or how much would they have } \\
\text { contributed to your decision on whether to } \\
\text { drink during pregnancy if they had discussed } \\
\text { it with you? }\end{array}$} & $\begin{array}{c}\text { Full } \\
\text { Sample }\end{array}$ & \multicolumn{2}{|c|}{ SPB } & \multicolumn{2}{|c|}{ NNR } & \multirow{2}{*}{$\begin{array}{c}\text { Strata } \\
\text { Difference } \\
\\
\\
J T^{\pi} \\
\text { (Z Statistic) } \\
\end{array}$} \\
\hline & Proportion & Pregnant & $\begin{array}{c}\text { Non- } \\
\text { pregnant }\end{array}$ & Pregnant & $\begin{array}{c}\text { Non- } \\
\text { pregnant }\end{array}$ & \\
\hline OB/GYN or nurse $\mathrm{e}^{\mathrm{a}, \mathrm{b}, \mathrm{c} 3}$ & & & & & & $-11.19 * *$ \\
\hline Not at all/Not very much/Not sure & 0.32 & 0.09 & 0.25 & 0.31 & 0.69 & \\
\hline Somewhat & 0.21 & 0.23 & 0.33 & 0.16 & 0.12 & \\
\hline A lot & 0.47 & 0.68 & 0.42 & 0.54 & 0.19 & \\
\hline Warning labels on bottles ${ }^{\mathrm{a}, \mathrm{b}, \mathrm{c} 2}$ & & & & & & $-9.65^{* *}$ \\
\hline Not at all/Not very much/Not sure & 0.58 & 0.38 & 0.53 & 0.00 & 0.84 & \\
\hline Somewhat & 0.34 & 0.49 & 0.43 & 0.00 & 0.10 & \\
\hline A lot & 0.08 & 0.14 & 0.04 & 0.00 & 0.06 & \\
\hline \multicolumn{7}{|l|}{ Newspapers/magazines } \\
\hline Not at all/Not very much/Not sure & 0.59 & 0.30 & 0.45 & 0.84 & 0.77 & \\
\hline Somewhat & 0.32 & 0.55 & 0.61 & 0.10 & 0.13 & \\
\hline A lot & 0.09 & 0.15 & 0.04 & 0.06 & 0.11 & \\
\hline \multicolumn{7}{|l|}{ Radio } \\
\hline Not at all/Not very much/Not sure & 0.61 & 0.33 & 0.48 & 0.85 & 0.78 & \\
\hline Somewhat & 0.30 & 0.53 & 0.48 & 0.10 & 0.11 & \\
\hline A lot & 0.09 & 0.14 & 0.04 & 0.06 & 0.11 & \\
\hline \multicolumn{7}{|l|}{ Television } \\
\hline Not at all/Not very much/Not sure & 0.57 & 0.29 & 0.44 & 0.82 & 0.74 & \\
\hline Somewhat & 0.32 & 0.54 & 0.50 & 0.11 & 0.11 & \\
\hline A lot & 0.12 & 0.17 & 0.06 & 0.07 & 0.16 & \\
\hline \multicolumn{7}{|l|}{ Posters/pamphlets in public places } \\
\hline Not at all/Not very much/Not sure & 0.64 & 0.34 & 0.46 & 0.89 & 0.88 & \\
\hline Somewhat & 0.30 & 0.54 & 0.49 & 0.08 & 0.08 & \\
\hline A lot & 0.06 & 0.12 & 0.05 & 0.03 & 0.04 & \\
\hline \multicolumn{7}{|l|}{ Posters/pamphlets in clinics } \\
\hline Not at all/Not very much/Not sure & 0.55 & 0.22 & 0.34 & 0.86 & 0.80 & \\
\hline Somewhat & 0.30 & 0.50 & 0.57 & 0.06 & 0.09 & \\
\hline A lot & 0.15 & 0.28 & 0.09 & 0.08 & 0.11 & \\
\hline \multicolumn{7}{|l|}{ Medical literature } \\
\hline Not at all/Not very much/Not sure & 0.45 & 0.13 & 0.27 & 0.69 & 0.76 & \\
\hline Somewhat & 0.28 & 0.38 & 0.48 & 0.14 & 0.12 & \\
\hline A lot & 0.17 & 0.49 & 0.25 & 0.17 & 0.12 & \\
\hline \multicolumn{7}{|l|}{ School } \\
\hline Not at all/Not very much/Not sure & 0.69 & 0.46 & 0.61 & 0.91 & 0.77 & \\
\hline Somewhat & 0.23 & 0.36 & 0.32 & 0.05 & 0.18 & \\
\hline A lot & 0.09 & 0.18 & 0.07 & 0.04 & 0.50 & \\
\hline Cost (too expensive) $)^{b, f}$ & & & & & & $-2.44 * *$ \\
\hline Not at all/Not very much/Not sure & 0.95 & 0.92 & 0.92 & 0.98 & 0.97 & \\
\hline Somewhat & 0.04 & 0.06 & 0.06 & 0.02 & 0.03 & \\
\hline A lot & 0.01 & 0.02 & 0.02 & 0.00 & 0.0 & \\
\hline \multicolumn{7}{|l|}{ Own experience } \\
\hline Not at all/Not very much/Not sure & 0.48 & 0.07 & 0.24 & 0.92 & 0.78 & \\
\hline Somewhat & 0.15 & 0.20 & 0.24 & 0.04 & 0.10 & \\
\hline A lot & 0.37 & 0.73 & 0.52 & 0.04 & 0.12 & \\
\hline Own knowledge/education ${ }^{\mathrm{a}, \mathrm{b}, \mathrm{f}}$ & & & & & & $-12.84 * *$ \\
\hline Not at all/Not very much/Not sure & 0.30 & 0.04 & 0.09 & 0.53 & 0.60 & \\
\hline Somewhat & 0.20 & 0.19 & 0.27 & 0.14 & 0.17 & \\
\hline A lot & 0.50 & 0.76 & 0.64 & 0.33 & 0.23 & \\
\hline
\end{tabular}

${ }^{\pi}$ Jonckheere-Terpstra test of ordered (mean response) differences between the four strata (two-sided test).

Significant main effects (reference group): ${ }^{\mathrm{a} P r e g n a n c y}$ status (non-pregnant), ${ }^{\mathrm{b}} \mathrm{City}$ (NNR), ${ }^{\mathrm{c} 2} \mathrm{~T}-\mathrm{ACE}$ (no risk), ${ }^{\mathrm{c} B}$ Binge drinking when nonpregnant (non-binge drinker), ${ }^{\mathrm{d}}$ Marital Status (single/divorced/never married/widowed), ${ }^{\mathrm{e}}$ Age ( $<30$ years), ${ }^{\mathrm{f}}$ Education ( $<$ middle level college)

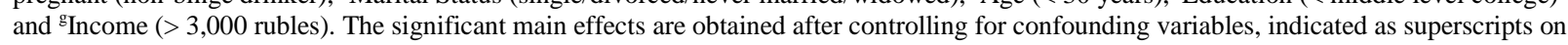


each question domain. For example, for the model examining differences in influence of husband/partner on decisions about drinking behavior (Husband/Partner, ${ }^{\mathbf{a}, \mathbf{b}, \mathbf{c}, \mathbf{c}, \mathbf{e}}$ ), the main effects reported for pregnancy status are adjusted for differences in city of residence, alcohol drinking risk captured using the T-ACE, binge drinking and age.

Reference category for the multinomial equations was the response category Not at all or Not very much or Not sure.

${ }^{* *} p$-value $<0.05,{ }^{*} p$-value $<0.10$ (two-tailed tests).

A total proportion of responses for each determinant equals $100 \%$ (or 1.0 )

Table 3

Importance of educating health professionals and community: Proportions of study participants' responses are indicated across four groups as defined by pregnancy status and city of residence.

\begin{tabular}{|c|c|c|c|c|c|c|}
\hline \multirow{2}{*}{$\begin{array}{l}\text { How important is it that the following people receive } \\
\text { information on drinking during pregnancy to help } \\
\text { you make a decision and/or support your decision } \\
\text { about drinking during pregnancy? }\end{array}$} & \multirow[b]{2}{*}{$\begin{array}{l}\text { Full Sample } \\
\text { Proportion }\end{array}$} & \multicolumn{2}{|c|}{ SPB } & \multicolumn{2}{|c|}{ NNR } & \multirow{2}{*}{$\begin{array}{c}\begin{array}{c}\text { Strata } \\
\text { Difference }\end{array} \\
T^{\pi} \\
\text { (Z Statistic) }\end{array}$} \\
\hline & & Pregnant & $\begin{array}{c}\text { Non- } \\
\text { pregnant }\end{array}$ & Pregnant & $\begin{array}{c}\text { Non- } \\
\text { pregnant }\end{array}$ & \\
\hline \multicolumn{7}{|l|}{ Physician or nurse } \\
\hline Not at all/Not very much/Not sure & 0.45 & 0.07 & 0.18 & 0.78 & 0.83 & \\
\hline Somewhat & 0.25 & 0.44 & 0.33 & 0.05 & 0.11 & \\
\hline A lot & 0.30 & 0.48 & 0.49 & 0.18 & 0.05 & \\
\hline OB/GYN or nurse $\mathrm{a}^{\mathrm{a}, \mathrm{b}}$ & & & & & & $-12.09 * *$ \\
\hline Not at all/Not very much/Not sure & 0.32 & 0.04 & 0.16 & 0.44 & 0.74 & \\
\hline Somewhat & 0.21 & 0.32 & 0.28 & 0.09 & 0.12 & \\
\hline A lot & 0.47 & 0.64 & 0.57 & 0.47 & 0.14 & \\
\hline Husband/partner ${ }^{\text {b,e }}$ & & & & & & $-11.84 * *$ \\
\hline Not at all/Not very much/Not sure & 0.38 & 0.09 & 0.13 & 0.66 & 0.68 & \\
\hline Somewhat & 0.26 & 0.38 & 0.38 & 0.10 & 0.15 & \\
\hline A lot & 0.37 & 0.53 & 0.49 & 0.24 & 0.16 & \\
\hline \multicolumn{7}{|l|}{ Best female friend } \\
\hline Not at all/Not very much/Not sure & 0.53 & 0.21 & 0.24 & 0.85 & 0.82 & \\
\hline Somewhat & 0.27 & 0.42 & 0.49 & 0.04 & 0.12 & \\
\hline A lot & 0.20 & 0.37 & 0.28 & 0.11 & 0.06 & \\
\hline Mother ${ }^{b, e}$ & & & & & & $-11.81 * *$ \\
\hline Not at all/Not very much/Not sure & 0.42 & 0.12 & 0.17 & 0.67 & 0.75 & \\
\hline Somewhat & 0.29 & 0.45 & 0.45 & 0.09 & 0.15 & \\
\hline A lot & 0.29 & 0.43 & 0.38 & 0.24 & 0.10 & \\
\hline \multicolumn{7}{|l|}{ Father } \\
\hline Not at all/Not very much/Not sure & 0.52 & 0.22 & 0.23 & 0.76 & 0.84 & \\
\hline Somewhat & 0.25 & 0.42 & 0.43 & 0.05 & 0.10 & \\
\hline A lot & 0.24 & 0.36 & 0.34 & 0.19 & 0.07 & \\
\hline Friends/co-workers ${ }^{\mathbf{b}}$ & & & & & & $-10.75^{* *}$ \\
\hline Not at all/Not very much/Not sure & 0.66 & 0.36 & 0.43 & 0.89 & 0.89 & \\
\hline Somewhat & 0.22 & 0.42 & 0.38 & 0.05 & 0.06 & \\
\hline A lot & 0.13 & 0.22 & 0.20 & 0.06 & 0.05 & \\
\hline \multicolumn{7}{|l|}{ Grandmother } \\
\hline Not at all/Not very much/Not sure & 0.65 & 0.36 & 0.49 & 0.92 & 0.90 & \\
\hline Somewhat & 0.19 & 0.36 & 0.29 & 0.02 & 0.06 & \\
\hline A lot & 0.16 & 0.28 & 0.23 & 0.06 & 0.05 & \\
\hline \multicolumn{7}{|l|}{ Grandfather } \\
\hline Not at all/Not very much/Not sure & 0.69 & 0.39 & 0.60 & 0.93 & 0.91 & \\
\hline Somewhat & 0.17 & 0.36 & 0.28 & 0.01 & 0.06 & \\
\hline A lot & 0.14 & 0.25 & 0.22 & 0.06 & 0.04 & \\
\hline
\end{tabular}

${ }^{\pi}$ Jonckheere-Terpstra test of ordered (mean response) differences between the four strata (two-sided test).

Significant main effects (Reference group): ${ }^{\mathrm{a}}$ Pregnancy status (non-pregnant), ${ }^{\mathrm{b}} \mathrm{City}$ (NNR), ${ }^{\mathrm{c} 1} \mathrm{TWE}$ AK risk (no risk), ${ }^{\mathrm{c} 2} \mathrm{~T}$-ACE (no risk), ${ }^{\mathrm{c} 3} \mathrm{Binge}$ drinking when non-pregnant (non-binge drinker), ${ }^{\mathrm{d}}$ Marital Status (single/divorced/never married/widowed), ${ }^{\mathrm{e}}$ Age ( $<30$ years), ${ }^{\mathrm{f}} \mathrm{Education}(<$ middle level college) and ${ }^{\mathrm{g}}$ Income ( $>$ 3,000 rubles). The significant main effects are obtained after controlling for confounding variables, indicated as superscripts on each question domain. For example, for the model examining differences in importance of OB/GYN or nurse education $\left(\mathbf{O B} / \mathbf{G Y N}\right.$ or Nurse $\left.{ }^{\mathbf{a}, \mathbf{b}}\right)$, the main effects reported for pregnancy status are adjusted for differences in city of residence. Reference category for the multinomial equations was the response category Not at all or Not very much or Not sure.

${ }^{* *} p$-value $<0.05,{ }^{*}$ p-value $<0.10$ (two-tailed tests). 


\section{Credibility of sources of information}

Study participants' responses about the credibility of different sources of information are reported in Table 4. The most credible message about drinking during pregnancy was a doctor's recommendation (A lot or Somewhat-85\%; 95\% CI: 59-100), followed closely by research data (A lot or Somewhat-84\%; 95\% CI: 58-100), and the least credible was a well-known spokesperson ( $A$ lot or Somewhat-50\%; 95\% CI: 30-78). There were significant differences in the proportion distributions among the four groups by pregnancy status and city in their respective responses about the credibility of research data, a doctor's recommendation, someone's personal testimony, and a well-known spokesperson's recommendation related to drinking during pregnancy $(p<.05)$.

Women with a higher level of education reported a greater belief in a doctor's $\left(\chi^{2}[2, N=620]=9.24, p<.05\right)$ or a well-known spokesperson's recommendation $\left(\chi^{2}[2, N=\right.$ $620]=8.94, p<.05)$ than women with lower education.
Women earning less than 3,000 rubles were more likely to believe a well-known person's recommendation or someone's personal testimony than were women earning more than 3,000 rubles, adjusting for education level, pregnancy status and city of residence $(p<.05)$.

Women at higher risk for drinking during pregnancy were more likely to believe personal testimony $\left(\chi^{2}[2, N=605]=\right.$ 11.12, $p<.05)$, while age had a negative association with tendency to believe personal testimony $\left(\chi^{2}[2, N=605]=\right.$ 7.37, $p<.05)$. Young women at higher risk of drinking during pregnancy were more likely to believe someone describing her or his experience than were their older counterparts who were at lower risk of alcohol use during pregnancy $(p<.05)$. Non-pregnant women involved in binge drinking reported a greater belief in research data $\left(\chi^{2}\right.$ [2, $N=628]=14.17, p<.05)$ and doctor's recommendations $\left(\chi^{2}[2, N=620]=9.03, p<.05\right)$ than did women not involved in binge drinking.

\section{Table 4}

Believability of different messaging contexts (Evaluative Statement 3): Proportions of study participants' responses across four groups defined by pregnancy status and city of residence.

\begin{tabular}{|c|c|c|c|c|c|c|}
\hline \multirow{2}{*}{$\begin{array}{l}\text { What kinds of information on drinking } \\
\text { during pregnancy would you believe? } \\
\text { How much would you believe it? }\end{array}$} & \multirow{2}{*}{$\begin{array}{c}\text { Full Sample } \\
\text { Proportion }\end{array}$} & \multicolumn{2}{|c|}{ SPB } & \multicolumn{2}{|c|}{ NNR } & \multirow{2}{*}{$\begin{array}{c}\begin{array}{c}\text { Strata } \\
\text { Difference }\end{array} \\
J T^{\pi} \\
\text { (Z Statistic) }\end{array}$} \\
\hline & & Pregnant & $\begin{array}{c}\text { Non- } \\
\text { pregnant }\end{array}$ & Pregnant & $\begin{array}{c}\text { Non- } \\
\text { pregnant }\end{array}$ & \\
\hline Research data $^{\text {a,b,c3 }}$ & & & & & & $-2.76 * *$ \\
\hline Not at all/Not very much/Not sure & 0.17 & 0.07 & 0.04 & 0.20 & 0.38 & \\
\hline Somewhat & 0.26 & 0.36 & 0.45 & 0.06 & 0.18 & \\
\hline Personal testimony ${ }^{\mathrm{a}, \mathrm{b}, \mathrm{c} 2, \mathrm{f}}$ & & & & & & $-5.00 * *$ \\
\hline Not at all/Not very much/Not sure & 0.36 & 0.11 & 0.13 & 0.73 & 0.52 & \\
\hline Somewhat & 0.33 & 0.46 & 0.5 & 0.12 & 0.20 & \\
\hline Very much & 0.31 & 0.43 & 0.37 & 0.15 & 0.28 & \\
\hline Doctor's recommendation ${ }^{\text {a,b,cs,f }}$ & & & & & & $-5.71 * *$ \\
\hline Not at all/Not very much/Not sure & 0.15 & 0.05 & 0.03 & 0.32 & 0.38 & \\
\hline Very much & 0.60 & 0.73 & 0.531 & 0.68 & 0.41 & \\
\hline Well-known spokesperson ${ }^{\text {a,b,f, g }}$ & & & & & & $-4.48 * *$ \\
\hline Not at all/Not very much/Not sure & 0.49 & 0.37 & 0.24 & 0.37 & 0.52 & \\
\hline Somewhat & 0.32 & 0.42 & 0.55 & 0.42 & 0.24 & \\
\hline Very much & 0.18 & 0.21 & 0.21 & 0.21 & 0.24 & \\
\hline
\end{tabular}

${ }^{\pi}$ Jonckheere-Terpstra test of ordered (mean response) differences between the four strata (two-sided test).

Significant main effects (Reference group): ${ }^{\mathrm{a}}$ Pregnancy status (non-pregnant), ${ }^{\mathrm{b}} \mathrm{City}(\mathrm{NNR}),{ }^{\mathrm{c} 1} \mathrm{TWEAK}$ risk (no risk), ${ }^{\mathrm{c} 2} \mathrm{~T}-\mathrm{ACE}$ (no risk), ${ }^{\mathrm{c}} \mathrm{Binge}$ drinking when non-pregnant (non-binge drinker), ${ }^{\mathrm{d}}$ Marital Status (single/divorced/never married/widowed), ${ }^{\mathrm{e}}$ Age $\left(<30\right.$ years), ${ }^{\mathrm{f}}$ Education (< middle level college) and ${ }^{g}$ Income (> 3,000 rubles). The significant main effects are obtained after controlling for confounding variables indicated

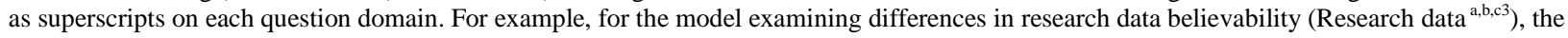
main effects reported for pregnancy status are adjusted for differences in city of residence and binge-drinking behavior.

Reference category for the multinomial equations was the response category Not at all or Not very much or Not sure.

** $p$-value $<0.05$, * $p$-value $<0.10$ (two-tailed tests). 


\section{Discussion}

This study demonstrates that the most influential factor on a woman's decision regarding alcohol consumption during pregnancy is her own knowledge; it thus provides evidence supporting the need for public education efforts in Russia. Women at both study locations reported that beliefs based on their "own knowledge" was the most influential factor in determining their drinking behavior, and this was more evident among pregnant women. The women's trust in research data possibly reflects the value attributed to science and higher education in Russian cultural traditions (Fedorov, 2011). Therefore, it is crucial that information offered in FASD prevention education is scientifically based and includes research data.

The study results show that Russian women hold OB/GYN physician and nurse recommendations in high regard and are more likely to believe them than information from other sources. It is important that physicians and nurses, particularly in OB/GYN clinics, are educated and skilled to help women in making decisions regarding their alcohol and contraception use; therefore, any health promotion efforts geared at FASD prevention in Russia should provide education for medical professionals. While education of the community as a whole may be an important universal prevention approach, this study's results indicate that some people who have been found to be influential in other populations, such as spouses or community members (Chang, McNamara, Orav, \& Wilkins-Haug, 2006), may have limited effects on Russian women's decisions related to alcohol use during pregnancy. While husbands, mothers, and coworkers or friends are influential for younger women, they have little relevance to decisions made by women ages 30 to 44; these women report relying more on their own education, on research data, and on medical professionals' recommendations. Women at greater risk for alcohol use during pregnancy are a particularly important target group for FASD prevention efforts. The study indicates that partners or husbands and warning labels may be influential in changing these women's drinking behavior during pregnancy, and young women who are at-risk drinkers are likely to benefit from hearing the personal testimony of others. Tailoring community education and warning labels to these groups of women is a promising approach. Similar to U.S. studies that found that higher alcohol prices negatively influence alcohol consumption among college students (Chaloupka, Grossman, \& Saffer, 2002; Wagenaar, Salois, \& Komro, 2009), this study indicates that increasing sale prices may be an effective strategy in reducing drinking among educated women in Russia.

Some limitations should be borne in mind when considering these findings. The analysis undertaken only examines what and how explanatory variables measured at the individual level affect the individual-level dependent variables (outcomes), and ignores how variables measured at the group level may have affected individual-level dependent variables. Future studies could explore the impact of group-level characteristics by looking at more than two geographical locations or clinics $(>30)$ to permit a number of units that would be sufficient to accommodate multi-level modeling. It was clear that there were differences between the SPB and NNR women's perceptions. The proportion of missing data across variables was minimal, as indicated in Table 1 , and therefore unlikely to affect study inferences under a complete case analysis approach. Under the assumption that data is missing completely at random, we believe our parameter estimates are unbiased.

Data was not obtained from women who declined study participation. This is also a potential limitation of the study, although it is unlikely to affect results because of the high study participation rate. As the two locations represent an inner city and a mix of urban and rural populations in Russia, the findings could vary at other locations. However, this contextual factor did not significantly moderate the effects of individual characteristics on any of the domains on the study questions about preferences, perceived credibility of information sources, and perceived importance of influencers' levels of knowledge. Although the tendency to trust research data might be amplified by social desirability bias, since respondents were part of this research study, it is also likely that it is a function of cultural traditions and reflects a more general tendency in Russia to value science and higher education.

The study results emphasize the importance of the dissemination of research data and education of health professionals, particularly OB/GYNs, in order to reduce alcohol consumption during pregnancy in Russia. Women's socio-demographic characteristics, including age and education, and their risk of alcohol consumption during pregnancy should be considered in designing prevention programs to achieve optimal effectiveness.

\section{Funding Sources}

This publication is supported by Grants R21TW006745 and R01AA016234 from the National Institutes of Health/National Institute on Alcohol Abuse and Alcoholism and Fogarty International Center (Brain Disorders in the Developing World: Research Across the Lifespan). The content is solely the responsibility of the authors and does not necessarily represent the official views of the National Institute on Alcohol Abuse and Alcoholism, Fogarty International Center, or the National Institutes of Health.

\section{Acknowledgments}

The investigators wish to thank the study consultants John Mulvihill, MD, Karen Beckman, MD, and Mark Wolraich, MD of OUHSC; Jacqueline Bertrand, Ph.D., of the Centers for Disease Control; Oleg Erishev, MD, of Bekhterev Research Institute; Edward Riley, Ph.D., of San Diego State University; and Alexander Palchik, MD and Vladimir Shapkaitz, MD, of St. Petersburg Pediatric Academy, and to acknowledge the contribution of staff and students who served on the project team from St. Petersburg State 
University, Nizhny Novgorod State Pedagogical University, and the University of Oklahoma Health Sciences Center.

\section{References}

American Academy of Pediatrics. Committee on substance abuse and committee on children with disabilities. Fetal alcohol syndrome and alcohol-related neurodevelopmental disorders. (2000). Pediatrics, 106, 358-361.

Atkin, C. K., \& Smith, S. W. (2010). Improving communication practices to reduce breast cancer environmental risks. Health Communication, 25, 587588. doi:10.1080/10410236.2010.496836

Balachova, T. N., Bonner, B. L., Isurina, G. L., \& Tsvetkova, L. A. (2007). Use of focus groups in developing FAS/FASD prevention in Russia. Substance Use \& Misuse, 42, 881-894.

Balachova, T., Bonner, B., Chaffin, M., Bard, D., Isurina, G., Tsvetkova, L., \& Volkova, E. (2012). Women's alcohol consumption and risk for alcohol-exposed pregnancies in Russia. Addiction, 107, 109-117.

Barry, K. L., Caetano, R., Chang, G., DeJoseph, M. C., Miller, L. A., O’Connor, M. J., Olson, H. C., Floyd, R. L., Weber, M. K., DeStefano, F., Dolina, S., \& Leeks, K. (2009). Reducing alcohol exposed pregnancies: A report of the National Task Force on Fetal Alcohol Syndrome and Fetal Alcohol Effect. Retrieved from Centers for Disease Control and Prevention: http://www.cdc.gov/ncbddd/fasd/documents/redalcohp reg.pdf

Centers for Diseases Control and Prevention. (2012). Alcohol use and binge drinking among women of childbearing age-United States, 2006-2010. Atlanta, GA. Morbidity and Mortality Weekly Report (MMWR), 61, 534-538.

Chaloupka, F. J., Grossman, M., \& Saffer, H. (2002). The effects of price on alcohol consumption and alcoholrelated problems. Alcohol Research \& Health, 26, 22 34.

Chambers, C. D., Kavteladze, L., Joutchenko, L., Bakhireva, L. N., \& Jones, K. L. (2006). Alcohol consumption patterns among pregnant women in the Moscow region of the Russian Federation. Alcohol, 38, 133-137.

Chang, G., McNamara, T. K., Orav, E. J., \& Wilkins-Haug, L. (2006). Alcohol use by pregnant women: partners, knowledge, and other predictors. Journal of Studies on Alcohol, 67, 245-251.

Ethen, M. K., Ramadhani, T. A., Scheherele, A. E., Canfield, M. A., Wyszynsji, D. F., Druschel, C. M., \& Romitti, P. A. (2007). National birth defects prevention study. Maternal Child Health, 13, 274-85.

Fedorov, V. (2011). Высшее образование в России: качество, ценность, востребованность. Пленарное заседание конференции «Образование и культура в процессе трансформации культуры» [Higher education in Russia: Quality, value, and demand]. Paper presented at the Conference on Education and
Culture in Process of Cultural Transformation, Moscow, Russia.

Floyd, R. L., Decoufle, P., \& Hungerford, D. W. (1999). Alcohol use prior to pregnancy recognition. American Journal of Preventive Medicine, 17, 101-107.

Floyd, R. L., Weber, M. K., Denny, C., \& O'Connor, M. J. (2009). Prevention of fetal alcohol spectrum disorders. Developmental Disabilities Research Reviews, 15, 193-199.

Foundation for Alcohol Research and Education. (2011). Alcohol Health Warning Labels: Attitudes and Perceptions. Retrieved from http://www.fare.org.au/ wp-content/uploads/2011/11/ FARE-Alcohol-HealthWarning-Labels-Attitudes-and-Perceptions_FINAL 1.pdf

Hankin, J. R. (2002). Fetal alcohol syndrome prevention research. [Review]. Alcohol Research \& Health, 26, 58-65.

Howat, P., Maycock, B., Cross, D., Collins, J., Jackson, L., Burns, S., \& James, R. (2003). Towards a more unified definition of health promotion. Health Promotion Journal of Australia, 14, 82-85. doi:10.1071/HE03082

Howat, P., Sleet, D., Elder, R., \& Maycock, B. (2004). Preventing alcohol-related traffic injury: a health promotion approach. Traffic Injury Prevention, 5, 208-219.

Interagency Coordinating Committee on Fetal Alcohol Spectrum Disorders (ICCFASD). (2011). Consensus statement on recognizing alcohol-related Neurodevelopmental Disorder (ARND) in primary health care of children. NIAAA: Rockville, MD. Retrieved from http://www.niaaa.nih.gov/sites/default/ files/ARNDConferenceConsensusStatementBooklet_ Complete.pdf

Kaskutas, L. A. (2000). Understanding drinking during pregnancy among urban American Indians and African Americans: health messages, risk beliefs, and how we measure consumption. Alcoholism: Clinical and Experimental Research, 24, 1241-1250.

Kesmodel, U., \& Olsen, S. F. (2001). Self reported alcohol intake in pregnancy: Comparison between four methods. Journal of Epidemiology \& Community Health, 55, 738-745.

Kesmodel, U., Kesmodel, P. S., Larsen, A., \& Secher, N. J. (2003). Use of alcohol and illicit drugs among pregnant Danish women, 1998. Scandinavian Journal of Public Health, 31, 5-11.

Kotler, P., \& Lee, N. (2008). Social marketing: Influencing behaviors for good. Thousand Oaks, CA, United States: Sage.

Kristjanson, A. F., Wilsnack, S. C., Zvartau, E., Tsoy, M., \& Novikov, B. (2007). Alcohol use in pregnant and non-pregnant Russian women. Alcoholism: Clinical and Experimental Research, 31, 299-307.

Landgren, M., Svensson, L., Stromland, K., \& Andersson Gronlund, M. (2010). Prenatal alcohol exposure and neurodevelopmental disorders in children adopted from eastern Europe. Pediatrics, 125, e1178-e1185.

Lum, M, Parvanta, C, Maibach, E, Arkin, E., \& Nelson, D. E. (2002). General public: Communicating to inform. In D. E. Nelson, R. C. Brownson, P. L. Remington, \& 
C. Parvanta (Eds.), Communicating public health information effectively: A guide for practitioners (pp. 47-57). Washington, DC, United States: American Public Health Association (APHA).

Miller, L. C., Chan, W., Litvinova, A., Rubin, A., Comfort, K., Tirella, L., . . . Kovalev, I. (2006). Fetal alcohol spectrum disorders in children residing in Russian orphanages: A phenotypic survey. Alcoholism: Clinical and Experimental Research, 30, 531-538.

National Institute on Alcohol Abuse and Alcoholism. (2004, Winter). NIAAA Council approves definition of binge drinking. NIAAA Newsletter, No. 3, 3.

National Institute on Alcohol Abuse and Alcoholism, (2005). Helping patients who drink too much: A clinician's guide. NIH publication No. 07-3769. Bethesda, MD, United States: National Institutes of Health, U.S. Department of Health and Human Services.

Nilsen, P., Holmqvist, M., Hultgren, E., Bendtsen, P., \& Cedergren, M. (2008). Alcohol use before and during pregnancy and factors influencing change among Swedish women. Acta Obstetrica et Gynecologica Scandinavica, 87, 768-774.

Onischenko, G. (2007). Об усилении надзора за производством и оборотом алкогольной продукцией. [Strengthening the supervision of the production and sale of alcohol products] Resolution No. 7, Moscow. Retrieved from http://rospotre bnadzor.ru/c/journal/view_article_content?groupId=10 156\&articleId $=82037 \&$ version $=1.0$

Palchik, A., Legonkova, S., \& Sofronova, G. (2011). Этнические особенности проявления алкогольного синдрома плода [Ethnic features of fetal alcohol syndrome manifestation]. Yakut Medical Journal, 34, 22-25.

Poole, N. A. (2008). Fetal Alcohol Spectrum Disorder (FASD) Prevention: Canadian perspectives. Public Health Agency of Canada. Retrieved from http://www.phac-aspc.gc.ca/hp-ps/dca-dea/progini/fasd-etcaf/publications/cp-pc/pdf/cp-pc-eng.pdf

Rasmussen, C., Kully-Martens, K., Denys, K., Badry, D., Henneveld, D., Wyper, K., \& Grant, T. (2012). The effectiveness of a community-based intervention program for women at risk for giving birth to a child with Fetal Alcohol Spectrum Disorder (FASD). Community Mental Health Journal, 48, 12-21.

Riley, E. P., Guerri, C., Calhoun, F., Charness, M. E., Foroud, T. M., Li, T.-K., . . . Warren, K. R. (2003). Prenatal alcohol exposure: Advancing knowledge through international collaborations. Alcoholism: Clinical and Experimental Research, 27, 118-135.

Russell, M. (1994). New assessment tools for risk drinking during pregnancy: T-ACE, TWEAK, and others. Alcohol Health \& Research World, 18, 55.

Russian Federation Federal State Statistics Service. (2009). Федеральная служба государственной статистики. [Federal State Statistics Services Website]. Retrieved from http://www.gks.ru/

SAS Institute Inc. (2008). SAS/STAT 9.2 User's Guide. Cary, NC, United States: SAS Institute Inc.
Siegel, M., \& Lotenberg, L. D. (2007). Marketing public health: Strategies to promote social change. Sudbury, MA, United States: Jones and Bartlett.

Skagerstrom, J., Chang, G., \& Nilsen, P. (2011). Predictors of drinking during pregnancy: a systematic review. Journal of Women's Health, 20(6), 901-913. doi:10.1089/jwh.2010.2216

Sokol, R. J., Martier, S. S., \& Ager, J. W. (1989). The TACE questions: practical prenatal detection of riskdrinking. American Journal of Obstetrics \& Gynecology, 160, 863-868.

Sprent, P. (2001). Applied nonparametric statistical methods. UK: Chapman \& Hall/CRC.

Sukhanova, L. P. (2008). Статистика родовспоможения как фактор обеспечения качества акушерской и перинатальной помощи в России. [Statistics of obstetric aid as a factor of providing quality obstetric and perinatal care in Russia]. "Социальные аспекты здоровья населения" [Social Aspects of Public Health], 4 (4). Retrieved from http://vestnik.mednet. $\mathrm{ru} /$ content/view/47/30/

Wagenaar, A. C., Salois, M. J., \& Komro, K. A. (2009). Effects of beverage alcohol price and tax levels on drinking: a meta-analysis of 1,003 estimates from 112 studies. Addiction, 104, 179-190.

Warren, K. R., Hewitt, B. G., \& Thomas, J. D. (2001). Fetal alcohol spectrum disorders: Research challenges and opportunities. Alcohol Research \& Health, 34, 414.

World Health Organization. (2011). Global Status Report on Alcohol and Health. Geneva: World Health Organization. Retrieved from http://www.who.int/ substance_abuse/publications/global_alcohol_report/m sbgsruprofiles.pdf

Yu, N., Ahern, L. A., Connolly-Ahern, C., \& Shen, F. (2010). Communicating the risks of fetal alcohol 